\title{
Local stability results for the collective behaviors of infinite populations of pulse-coupled oscillators
}

\author{
Alexandre Mauroy and Rodolphe Sepulchre
}

\begin{abstract}
In this paper, we investigate the behavior of pulsecoupled integrate-and-fire oscillators. Because the stability analysis of finite populations is intricate, we investigate stability results in the approximation of infinite populations. In addition to recovering known stability results of finite populations, we also obtain new stability results for infinite populations. In particular, under a weak coupling assumption, we solve for the continuum model a conjecture still prevailing in the finite dimensional case.
\end{abstract}

\section{INTRODUCTION}

In a seminal work on pacemaker cells of the heart, Peskin introduced a model of (identical) pulse-coupled Leaky Integrate-and-Fire (LIF) oscillators, characterized by a monotone dynamics [1]. Two complementary studies [2], [3] provided a complete global stability analysis of the model and highlighted an interesting dichotomy: the oscillators either achieve perfect synchrony or converge to an asynchronous behavior.

Generalizations of the original LIF model have become popular in the recent years as reduced models of realistic models of neurons, see e.g. [4]. The Quadratic Integrate-andFire (QIF) model is an important such example, for which the monotonicity assumptions of the original LIF model no longer hold. Surprisingly, the dichotomic behavior proven for the LIF model seems to persist in the QIF model and other generalizations. But even a local stability analysis has proven elusive so far, see e.g. [5].

In this paper, we address this stability question by considering the continuum limit of a large number of pulsecoupled oscillators. In the case of a weak coupling, we solve the conjecture on QIF oscillators prevailing in the original model.

The paper is organized as follows. In Section II, we introduce the model of pulse-coupled oscillators and the continuum approximation. The dichotomic behavior observed with monotone dynamics is described in Section III and the problems we face with non-monotone dynamics are presented in Section IV. Section V provides a general stability analysis of the continuum model and Section VI presents our main results on local stability of weakly coupled oscillators. In the case of non-monotone dynamics, a non-dichotomic behavior is described in Section VII. Finally, we give some concluding remarks in Section VIII.

A. Mauroy and R. Sepulchre are with the Department of Electrical Engineering and Computer Science, University of Liège, 4000 Liège, Belgium, alexandre.mauroy@ulg.ac.be, r. sepulchre@ulg.ac.be

\section{Preliminaries}

We present a brief overview of pulse-coupled oscillators and the phase density model obtained in the case of infinite populations.

\section{A. Models of pulse-coupled oscillators}

In this paper, we consider models of integrate-and-fire oscillators [6]. An integrate-and-fire oscillator is described by a scalar state variable $x$, which monotonically increases between the two thresholds $\underline{x}$ and $\bar{x}$ according to the dynamics $\dot{x}=F(x), F>0$. When the oscillator reaches the upper threshold $\bar{x}$, it is reset to the lower threshold $\underline{x}$ (it is said to fire)

As initially proposed in [1], the coupling between $N$ integrate-and-fire oscillators within the network is impulsive. Whenever an oscillator fires, it sends out a pulse which instantaneously increments the state of all the other oscillators by a constant value $K / N$, where $K$ is the coupling strength. The coupling is usually excitatory $(K>0)$ but may also be inhibitory $(K<0)$. The dynamics of a pulse-coupled integrate-and-fire oscillator $i \in\{1, \ldots, N\}$ is then given by

$$
\dot{x}_{i}=F\left(x_{i}\right)+u_{i}(t),
$$

with the coupling

$$
u_{i}(t)=\frac{K}{N} \sum_{\substack{j=1 \\ j \neq i}}^{N} \sum_{k=0}^{\infty} \delta\left(t-t_{k}^{(j)}\right) .
$$

The Dirac functions $\delta$ model the pulses which increment the state of oscillator $i$ at the firing times $t_{k}^{(j)}$, that is, when an oscillator $j \neq i$ fires.

For the sake of simplicity, the dynamics of the oscillators is turned into a phase dynamics. The phase $\theta \in S^{1}(0,2 \pi)$ is determined from the state $x$ by rescaling in such a way that $\theta=0$ corresponds to the low threshold $x=\underline{x}$ and that a single (uncoupled) oscillator satisfies $\dot{\theta}=\omega$, where $\omega$ is the natural frequency of the oscillator. The state-phase relation is given by

$$
\theta=\omega \int_{\underline{x}}^{x} \frac{1}{F\left(x^{\prime}\right)} d x^{\prime} .
$$

The state dynamics (1) of oscillator $i$ is rewritten as the phase dynamics

$$
\dot{\theta}_{i}=\omega+Q\left(\theta_{i}\right) u_{i}(t) \triangleq v\left(\theta_{i}, t\right),
$$

where $Q:[0,2 \pi] \mapsto \mathbb{R}$ is the phase response curve (PRC) of the oscillator, that is, the phase sensitivity of the oscillator to an external perturbation (in this case, the coupling) [4], 
[7]. For integrate-and-fire oscillators, the PRC has the exact analytical expression (see [8])

$$
Q(\theta)=\frac{\omega}{F[x(\theta)]} .
$$

\section{B. Phase density model}

For large networks, a good approximation is to consider an infinite number of oscillators. In this case, a continuum of oscillators is described by a density function which evolves according to a nonlinear partial differential equation (PDE).

a) Phase density equation: In the limit of an infinite number of oscillators, the population is a continuum characterized by a phase density function $\rho(\theta, t)$ normalized on $S^{1}(0,2 \pi)$. Defining the flux as

$$
J(\theta, t)=\rho(\theta, t) v(\theta, t),
$$

the evolution of the oscillators obeys the continuity equation

$$
\frac{\partial}{\partial t} \rho(\theta, t)=-\frac{\partial}{\partial \theta} J(\theta, t) .
$$

Since the phase $\theta$ is defined on $S^{1}(0,2 \pi) \equiv \mathbb{R} \bmod 2 \pi$, the PDE verifies the boundary condition

$$
J(0, t)=J(2 \pi, t) \triangleq J_{0}(t) \quad \forall t .
$$

For the sake of simplicity, we use in the sequel the notation $J_{0}$ to denote the boundary flux (7).

b) Impulsive coupling: For a finite population, the oscillators crossing $\theta=0$ at times $t_{k}^{(j)}$ induce a flux $J_{0}(t)=\frac{1}{N} \sum_{j} \sum_{k} \delta\left(t-t_{k}^{(j)}\right)$. In the limit $N \rightarrow \infty$, the influence of a single oscillator is negligible, so that comparing the flux $J_{0}$ with the coupling (2) yields the coupling $u(t)=K J_{0}(t)$, which is common to the whole population. The coupling is a continuous-time function interpreted as an infinite sum of infinitesimal spikes (of size $K / N \ll 1$ ). For the continuum of pulse-coupled oscillators, the phase dynamics (4) then writes

$$
v(\theta, t)=\omega+Q(\theta) K J_{0}(t) .
$$

\section{Dichotomy IN THE LIF MODEL}

Impulsive coupling was initially studied in [1] for leaky integrate-and-fire (LIF) oscillators, characterized by the monotone dynamics $\dot{x}=F(x)=S+\gamma x>0$ $\forall x \in[\underline{x}, \bar{x}]=[0,1]$. Using the monotonicity property of the dynamics, two complementary studies showed that finite populations of pulse-coupled LIF oscillators exhibit two contrasting behaviors. The two studies thereby highlighted that the behavior is characterized by an interesting dichotomy. If $K d F / d x<0 \forall x \in[\underline{x}, \bar{x}]$, the oscillators achieve perfect synchrony for almost all initial conditions [2]. If $K d F / d x>0 \forall x \in[\underline{x}, \bar{x}]$, the oscillators globally converge to a phase-locked clustering configuration [3]. In the latter situation, the clusters spread over the circle $S^{1}(0,2 \pi)$ so that the behavior is an asynchronous state.

Our recent work [9] shows that the dichotomic behavior of LIF oscillators also prevails in the continuum limit of the model. In Fig. 1, the oscillators either perfectly synchronize or converge toward an asynchronous state. In case of synchronization, the flux $J_{0}$ tends to a series of Dirac functions, which shows that the whole population concurrently crosses the threshold $\theta=0$. In case of asynchronous state, the flux converges to the uniform (constant) flux $J^{*}$. Since the coupling has a repulsive effect which spreads the oscillators over the circle $S^{1}(0,2 \pi)$, this behavior is the continuous equivalent of the phase-locked asynchronous behavior observed for finite populations.
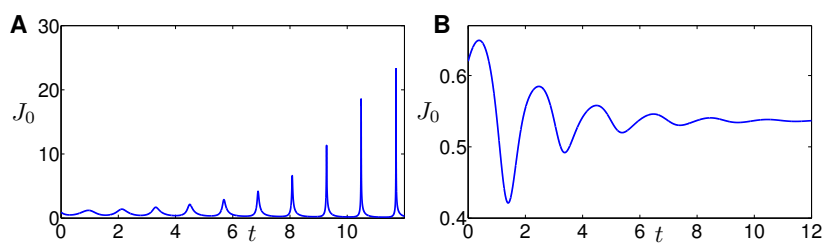

Fig. 1. The infinite population of LIF oscillators has a dichotomic behavior. A. When $K d F / d x<0$, the oscillators achieve perfect synchrony: the flux $J_{0}$ tends to a series of Dirac functions. B. When $K d F / d x>0$, the density converges toward an asynchronous state: the flux tends to the uniform flux $J^{*} \approx 0.53$. (The simulations are performed with a finite differences method, with $F(x)=2.1-2 x$ and $K= \pm 0.1$.)

Dichotomy is a global property of the model. For oscillators with a monotone dynamics, the global analysis of the dichotomy relies on the existence of a Lyapunov function, which shows the global stability (or instability) of the asynchronous state for finite populations [3] and for infinite populations [9].

\section{BEYOND MONOTONICITY ASSUMPTIONS}

Even though the LIF model is the most popular integrateand-fire model, its monotone dynamics implies that the time evolution of the oscillators has a curvature with a constant sign. Such a dynamics is not representative of various biological oscillators (such as neurons) and considering more complex (non-monotone) dynamics is a relevant problem.

For instance, an important generalization of the LIF model is the quadratic integrate-and-fire (QIF) model, defined by $F(x)=S+x^{2}, S>0$ [10]. Numerical simulations suggest clear evidence that pulse-coupled QIF oscillators have also a dichotomic behavior. The simulations show that the behavior is determined by the average derivative $K d F / d x$ between the two thresholds, that is, by the quantity

$$
K[F(\bar{x})-F(\underline{x})] .
$$

Numerical evidence suggests that QIF oscillators perfectly synchronize when (9) is negative and converge to the asynchronous state when (9) is positive.

In contrast to monotone dynamics, there is no rigorous proof of dichotomy in the QIF model. Whether the asynchronous state is globally stable is an open problem so far. More surprisingly, our recent study [5] on finite populations has revealed that even a local stability analysis is an intricate problem, yielding the following conjecture:

Conjecture 1: For the QIF model $F(x)=S+x^{2}$, the asynchronous state is locally stable if and only if $K[F(\bar{x})-F(\underline{x})]>0$. 
To get a deeper insight into the problem, we consider other "QIF-like" integrate-and-fire dynamics satisfying the following assumption.

Assumption 1: $F(\cdot):[\underline{x}, \bar{x}] \mapsto \mathbb{R}$ is continuous, positive, even, and strictly increasing on $[0, \bar{x}]$.

Representative models of this class are the exponential model, defined by $F(x)=S \exp \left(x^{2}\right), S>0$, and the piecewise linear model, that is, a direct generalization of the linear LIF model defined by $F(x)=S+\gamma|x|, S, \gamma>0$ (Fig. 2).



Fig. 2. Three models (quadratic, exponential, and piecewise linear) satisfying Assumption 1. The behavior of the oscillators differs when $K[F(\bar{x})-F(\underline{x})]>0$ or $K[F(\bar{x})-F(\underline{x})]<0$.

Whereas local stability analysis of the QIF model is an open question, a (fastidious) local analysis is provided in [5] for finite populations of exponential and piecewise linear oscillators. Interestingly, the analysis shows that the dichotomy is not an obvious and inherent characteristic of pulse-coupled oscillators: while the local analysis supports the dichotomic behavior in the exponential model, the study shows that the dichotomy does not persist in the piecewise-linear model. In this context, the QIF model, with its conjecture, is an intermediate case between the exponential model and the piecewise-linear model.

The analysis of models satisfying Assumption 1 is much more involved than the analysis of models with a monotone dynamics. The stability problem, however local or not, is complex and yields some open problems in the case of finite populations. For these reasons, we investigate in the rest of the paper the continuum approximation of the models and we present further stability results, including the proof of Conjecture 1 in the weak coupling limit.

\section{LOCAL STABILITY ANALYSIS OF THE CONTINUUM MODEL}

In the continuum limit, we investigate the local stability of the asynchronous state (Fig. 1B). The asynchronous state corresponds to the stationary solution of (6)-(8):

$$
J(\theta)=J^{*}, \quad \rho^{*}(\theta)=\frac{J^{*}}{\omega+K Q(\theta) J^{*}} .
$$

This solution exists if there exists a value $J^{*}$ so that the stationary density (10) is normalized on $[0,2 \pi]$. In [9], we give a necessary and sufficient condition for the existence of the stationary solution.

To study the local stability of the stationary asynchronous state, we linearize the continuity equation (6)-(8) around the stationary solution (10) and we obtain the eigenvalue equation (see Appendix)

$$
e^{\lambda / J^{*}}-1=\frac{K \lambda}{4 \pi^{2} J^{*}} \int_{0}^{2 \pi} \Gamma(\vartheta) e^{\lambda \vartheta /\left(2 \pi J^{*}\right)} d \vartheta
$$

For the sake of simplicity, a new phase variable has been introduced, which satisfies

$$
\frac{d \vartheta}{d \theta}=\frac{2 \pi J^{*}}{\omega+K Q(\theta) J^{*}},
$$

so that the oscillators have a constant velocity $\dot{\vartheta}=2 \pi J^{*}$ under the asynchronous regime $J_{0}=J^{*}$ (see (18)). In addition, $\vartheta=0$ (resp. $\vartheta=2 \pi$ ) corresponds to $\theta=0$ (resp. $\theta=2 \pi)$. The function

$$
\Gamma(\vartheta)=\frac{d \vartheta}{d \theta} Q(\theta)=\frac{2 \pi J^{*} Q(\theta)}{\omega+J^{*} K Q(\theta)}
$$

is interpreted as a PRC related to phase $\vartheta$ and corresponds to the oscillators phase sensitivity to a perturbation of the asynchronous regime.

The eigenvalue equation (11) has an infinity of solutions $\lambda_{n}(n \in \mathbb{Z})$ whose real parts determine the stability of the asynchronous state. When the real parts of the eigenvalues are all negative, the asynchronous state is locally stable. When they are all positive, all the modes are unstable and the flux tends to a Dirac function. In the uncoupled case $K=0$, the eigenvalues $\lambda_{n}=i n \omega$ all lie on the imaginary axis and the asynchronous state is marginally stable.

Whether the model satisfies Assumption 1 or not, the whole set of eigenvalues can only be obtained numerically. However, some properties of the eigenvalues, summarized in the following proposition, provide useful insight in the stability analysis.

Proposition 1: $1.0 \triangleq \lambda_{0}$ is a solution of (11);

2. If $\lambda_{n}$ is a solution of (11), then its complex conjugate $\bar{\lambda}_{n} \triangleq \lambda_{-n}$ is also a solution;

3. There exist solutions satisfying $\Im\left\{\lambda_{n}\right\} \rightarrow \infty$ which tend to

$$
\lambda_{\infty} \triangleq J^{*} \log \frac{\omega+J^{*} K Q(2 \pi)}{\omega+J^{*} K Q(0)}+i n 2 \pi J^{*} \quad(n \rightarrow \infty) .
$$

Proof: The first two points are trivial. The latter is proven as follows. Integrating by parts the right hand of (11) yields

$$
\begin{aligned}
e^{\lambda / J^{*}}-1= & \frac{K}{2 \pi}\left[\Gamma(2 \pi) e^{\lambda / J^{*}}-\Gamma(0)\right] \\
& -\frac{K}{2 \pi} \int_{0}^{2 \pi} \frac{d \Gamma}{d \vartheta} e^{\lambda \vartheta /\left(2 \pi J^{*}\right)} d \vartheta .
\end{aligned}
$$

Considering that $\Im\{\lambda\} \rightarrow \infty$, the Riemann-Lebesgue lemma [11] implies that the integral in (14) vanishes. Then it follows from (13) that (14) writes

$$
e^{\lambda / J^{*}}=\frac{\omega+J^{*} Q(2 \pi)}{\omega+J^{*} Q(0)} .
$$

The solution of this equation is $\lambda_{\infty}$, which concludes the proof. 
Proposition 1 provides information for the eigenvalues corresponding to high frequency modes. The real part of these eigenvalues asymptotically tends to the value $\Re\left\{\lambda_{\infty}\right\}$. The value has the same sign as $K[Q(2 \pi)-Q(0)]$, or given (5), as $-K[F(\bar{x})-F(\underline{x})]$. The high frequency modes are thus stable if (9) is positive. Since eigenvalues of lower frequency can have real parts of opposite sign, the condition on (9) is only a necessary condition for the stability of the asynchronous state but it highlights the role of the quantity (9) as a stability criterion.

The key role of (9) on the stability is reinforced for models satisfying Assumption 1. In this case, the symmetry property of the dynamics induces a PRC with a reflection symmetry depending on (9):

Property 1: A model satisfying Assumption 1 has a PRC

$$
Q(\theta)=Q_{e}(\theta-\bar{\theta}) \quad \forall \theta \in[0,2 \pi]
$$

where $Q_{e}(\cdot)$ is an even function on $\mathbb{R}$. To the inequality $F(\bar{x})-F(\underline{x})>0$ (resp. $F(\bar{x})-F(\underline{x})<0$ ) corresponds the inequality $\bar{\theta}<\pi$ (resp. $\bar{\theta}>\pi$ ). In addition, changing the sign of $F(\bar{x})-F(\underline{x})$ yields a mirrored PRC, that is, it modifies the symmetry point $\bar{\theta}$, which becomes $2 \pi-\bar{\theta}$.

Property 1 directly follows from (3) and (5). As shown in the following proposition, Property 1 is of interest for the stability analysis.

Proposition 2: Let $Q^{(1)}$ and $Q^{(2)}$ denote two PRC which satisfy $Q^{(1)}(\theta)=Q^{(2)}(2 \pi-\theta)$. Then, the respective eigenvalues, solutions of (11), satisfy $\Re\left\{\lambda_{n}^{(1)}\right\}=-\Re\left\{\lambda_{n}^{(2)}\right\}$. In particular, for a pulse-coupled model verifying Assumption 1 , changing the sign of $F(\bar{x})-F(\underline{x})$ changes the sign of the real parts $\Re\left\{\lambda_{n}\right\}$.

Proof: Given the hypothesis, it follows from (12) and (13) that $\Gamma^{(1)}(\vartheta)=\Gamma^{(2)}(2 \pi-\vartheta)$. In addition, one easily shows that the stationary flux $J^{*}$ is the same for the two PRC. Then, the eigenvalue equation (11) for $\Gamma^{(1)}$ can be rewritten as

$$
e^{\lambda / J^{*}}-1=\frac{K \lambda}{4 \pi^{2} J^{*}} \int_{0}^{2 \pi} \Gamma^{(2)}(2 \pi-\vartheta) e^{\lambda \vartheta /\left(2 \pi J^{*}\right)} d \vartheta
$$

or

$$
e^{-\lambda / J^{*}}-1=\frac{K(-\lambda)}{4 \pi^{2} J^{*}} \int_{0}^{2 \pi} \Gamma^{(2)}(\vartheta) e^{-\lambda \vartheta /\left(2 \pi J^{*}\right)} d \vartheta
$$

If $\lambda_{n}^{(1)}$ is an eigenvalue for $\Gamma^{(1)}$, then $-\lambda_{n}^{(1)} \triangleq \lambda_{-n}^{(2)}$ is an eigenvalue for $\Gamma^{(2)}$. Since $\lambda_{-n}^{(2)}=\bar{\lambda}_{n}^{(2)}$, one has $\Re\left\{\lambda_{n}^{(1)}\right\}=-\Re\left\{\lambda_{n}^{(2)}\right\}$. When Assumption 1 is satisfied, the second part of the proposition follows from Property 1.

Proposition 2 shows how models satisfying Assumption 1 are the most likely to exhibit dichotomy: two opposite values $F(\bar{x})-F(\underline{x})$ (corresponding to two mirrored PRC) yield two behaviors with opposite stability.

Remark 1: The particular case $F(\bar{x})-F(\underline{x})=0$ corresponds to a marginally stable behavior. In this case, one has $\bar{\theta}=\pi$ and the oscillators have a symmetric PRC $Q(\theta)=Q(2 \pi-\theta)$. Given the resulting symmetry of
$\Gamma(\vartheta)=\Gamma_{e}(\vartheta-\pi)$, with $\Gamma_{e}(\cdot)$ an even function, the eigenvalue equation (11) writes

$$
e^{\lambda /\left(2 J^{*}\right)}-e^{-\lambda /\left(2 J^{*}\right)}=\frac{K \lambda}{4 \pi^{2} J^{*}} \int_{-\pi}^{\pi} \Gamma_{e}(\vartheta) e^{\lambda \vartheta /\left(2 \pi J^{*}\right)} d \vartheta .
$$

Assuming a purely imaginary solution $\lambda=i|\lambda|$, the equation becomes

$$
\sin \left(\frac{|\lambda|}{2 J^{*}}\right)=\frac{K|\lambda|}{4 \pi^{2} J^{*}} \int_{0}^{\pi} \Gamma_{e}(\vartheta) \cos \left(\frac{|\lambda| \vartheta}{2 \pi J^{*}}\right) d \vartheta
$$

and has an infinity of solutions. A symmetric PRC $Q(\theta)=Q(2 \pi-\theta)$ is a necessary condition to ensure an infinity of eigenvalues on the imaginary axis.

\section{LOCAL STABILITY RESULTS FOR WEAKLY COUPLED OSCILLATORS}

The preceding section provides qualitative properties derived from the eigenvalue equation but does not provide a full local stability analysis. In contrast, an analytical characterization of the eigenvalues becomes available when the coupling is weak. Using a simple stability criterion, we will recover stability results known for finite populations and we will prove Conjecture 1.

When $K \ll 1$, the stationary density (10) is approximated by a constant $\rho^{*} \approx J^{*} / \omega$ and $2 \pi J^{*} \approx \omega$ since $\rho^{*}$ is normalized on $[0,2 \pi]$. Then, (12) implies $\vartheta \approx \theta$ and (13) leads to $\Gamma(\vartheta) \approx Q(\theta)$. It follows that the eigenvalue equation (11) is simplified to

$$
e^{(2 \pi \lambda) / \omega}-1=\frac{K \lambda}{2 \pi \omega} \int_{0}^{2 \pi} Q(\theta) e^{\lambda \theta / \omega} d \theta .
$$

Next, linearizing the equation around the solutions $\lambda_{n}=i n \omega$ of the case $K=0$ leads to

$$
\Re\left\{\lambda_{n}\right\}=-\frac{K n \omega}{4 \pi^{2}} \int_{0}^{2 \pi} Q(\theta) \sin (n \theta) d \theta+\mathcal{O}\left(K^{2}\right) .
$$

Equality (16) can also be expressed as

$$
\Re\left\{\lambda_{n}\right\}=\frac{K n \omega}{2 \pi} \Im\left\{\hat{Q}_{n}\right\}+\mathcal{O}\left(K^{2}\right),
$$

with $\hat{Q}_{n}$ the nth Fourier coefficient of the PRC

$$
\hat{Q}_{n}=\frac{1}{2 \pi} \int_{0}^{2 \pi} Q(\theta) e^{-i n \theta} d \theta .
$$

The expression (17) is in fact not new. It was used in [12], [13] to study the local stability of weakly coupled LIF oscillators. However, (16) gives an explicit expression of the eigenvalues in the general case. In the sequel, we use this result to study the local stability of models satisfying Assumption 1.

QIF model: In the case of a weak coupling, the following proposition proves Conjecture 1 .

Proposition 3: For a continuum of weakly pulse-coupled QIF oscillators, the asynchronous state is locally stable if and only if $K[F(\bar{x})-F(\underline{x})]>0$.

Proof: Given (5), the PRC of QIF oscillators expresses as

$$
Q(\theta)=\frac{\omega}{S} \cos ^{2}\left[\frac{\sqrt{S}}{\omega}(\theta-\bar{\theta})\right]
$$


with

$$
\omega=2 \pi \sqrt{S} /\left[\arctan \frac{\bar{x}}{\sqrt{S}}-\arctan \frac{\underline{x}}{\sqrt{S}}\right]
$$

and

$$
\bar{\theta}=-2 \pi \frac{\arctan (\underline{x} / \sqrt{S})}{\arctan (\bar{x} / \sqrt{S})-\arctan (\underline{x} / \sqrt{S})} .
$$

Assuming $K \neq 0$, (16) leads to

$$
\frac{\Re\left\{\lambda_{n}\right\}}{K}=\frac{n^{2} \omega^{4} \sin \left(4 \pi^{2} \sqrt{S} / \omega\right)}{2 \pi S\left(\omega^{2} n^{2}-4 S\right)} \sin \left[\frac{2 \sqrt{S}}{\omega}(\bar{\theta}-\pi)\right] .
$$

One easily shows that $\omega>2 \sqrt{S}$ and $2 \sqrt{S}|\bar{\theta}-\pi| / \omega<\pi$. Then, it follows that $\Re\left\{\lambda_{n}\right\} / K<0 \forall n \neq 0$ if $\bar{\theta}<\pi$ and that $\Re\left\{\lambda_{n}\right\} / K>0 \forall n \neq 0$ if $\bar{\theta}>\pi$. The relation between $\bar{\theta}$ and $F(\bar{x})-F(\underline{x})$ (see Property 1 ) concludes the proof.

For a continuum of weakly coupled oscillators, Conjecture 1 is proven. In the sequel, we also recover all the results known in the case of finite populations, which validates the continuum limit approximation.

Exponential model: Our previous study [5] provides a stability result for finite populations of exponential integrateand-fire oscillators. For the continuum under weak coupling, a similar stability result is obtained, which complements the previous result.

Exponential integrate-and-fire oscillators have a concavedown PRC. Then, the local stability of these oscillators is characterized by the following proposition.

Proposition 4: Consider a continuum of weakly pulsecoupled integrate-and-fire oscillators which (1) satisfy Assumption 1 and (2) have a PRC satisfying $d^{2} Q / d \theta^{2}<0$ $\forall \theta \in[0,2 \pi]$. Then, the asynchronous state is locally stable if and only if $K[F(\bar{x})-F(\underline{x})]>0$.

Proof: Since the model satisfies Assumption 1, the PRC can be rewritten as (15), with $d^{2} Q_{e} / d \theta^{2}<0$. Using (16), one computes the variation of $\Re\left\{\lambda_{n}\right\}(n \neq 0)$ corresponding to a variation of the symmetry point $\bar{\theta}$. Assuming $K \neq 0$, this yields

$$
\begin{aligned}
& \frac{d}{d \bar{\theta}} \Re\left\{\lambda_{n}\right\} / K \\
& =\left.\frac{n \omega}{4 \pi^{2}} \int_{0}^{2 \pi} \sin (n \theta) \frac{d Q_{e}}{d \theta}\right|_{\theta-\bar{\theta}} d \theta \\
& =\frac{\omega}{4 \pi^{2}}\left(\left.\frac{d Q_{e}}{d \theta}\right|_{-\bar{\theta}}-\left.\frac{d Q_{e}}{d \theta}\right|_{2 \pi-\bar{\theta}}+\left.\int_{0}^{2 \pi} \cos (n \theta) \frac{d^{2} Q_{e}}{d \theta^{2}}\right|_{\theta-\bar{\theta}} d \theta\right) \\
& >\frac{\omega}{4 \pi^{2}}\left(\left.\frac{d Q_{e}}{d \theta}\right|_{-\bar{\theta}}-\left.\frac{d Q_{e}}{d \theta}\right|_{2 \pi-\bar{\theta}}+\left.\int_{0}^{2 \pi} \frac{d^{2} Q_{e}}{d \theta^{2}}\right|_{\theta-\bar{\theta}} d \theta\right)=0 .
\end{aligned}
$$

When $\bar{\theta}=\pi$, one has $\Re\left\{\lambda_{n}\right\}=0$ (see Remark 1). Then, it follows that $\Re\left\{\lambda_{n}\right\} / K<0 \forall n \neq 0$ if $\bar{\theta}<\pi$ and $\Re\left\{\lambda_{n}\right\} / K>0 \forall n \neq 0$ if $\bar{\theta}>\pi$. The relation between $\bar{\theta}$ and $F(\bar{x})-F(\underline{x})$ (see Property 1 ) concludes the proof.

For exponential integrate-and-fire oscillators, Proposition 5 implies that the local stability of the asynchronous state is characterized by the quantity (9). The result is in good agreement with the result obtained for finite populations.
Piecewise-linear model: Our previous study [5] shows that piecewise linear integrate-and-fire oscillators are not characterized by a dichotomic behavior. The PRC of piecewise linear oscillators is

$$
Q(\theta)=\frac{\omega}{S} \exp \left(-\frac{\gamma}{\omega}|\theta-\bar{\theta}|\right)
$$

with

$$
\omega=2 \pi \gamma / \log \left[\frac{(S-\gamma \underline{x})(S+\gamma \bar{x})}{S^{2}}\right]
$$

and

$$
\bar{\theta}=\frac{2 \pi \log [(S-\gamma \underline{x}) / S]}{\log [(S-\gamma \underline{x}) / S]+\log [(S+\gamma \bar{x}) / S]} .
$$

The relation (16) leads to

$$
\begin{array}{r}
\Re\left\{\lambda_{n}\right\}=\frac{K n^{2} \omega^{3}}{2 \pi^{2} S\left(\gamma^{2}+n^{2} \omega^{2}\right)}\left\{-\frac{\gamma}{n} \sin (n \bar{\theta})\right. \\
\left.+\omega e^{-\pi \gamma / \omega} \sinh \left[\frac{\gamma}{\omega}(\bar{\theta}-\pi)\right]\right\} .
\end{array}
$$

The second term of the right hand, which dominates for $n \gg 1$, has a constant sign for all $n$. However, the first term is not negligible for lower values $n$ and has a sign which depends on $n$. For some parameters, the values $\Re\left\{\lambda_{n}\right\}$ with a low $n$ can be both positive and negative, depending on $n$ (see Fig. 3). In this situation, there is always at least one unstable eigenvalue (independently of the sign of (9)). As in the case of a finite population, the behavior is not dichotomic.

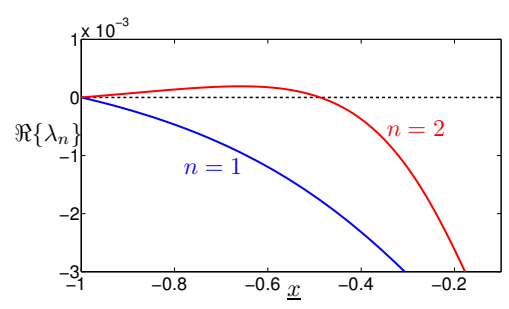

Fig. 3. Piecewise linear oscillators have not a dichotomic behavior. When $\underline{x}<-0.5$, one has $\Re\left\{\lambda_{2}\right\}>0$ while $\Re\left\{\lambda_{1}\right\}<0$. (The parameters are $\bar{S}=1, \gamma=1, K=0.510^{-2}$, and $\bar{x}=1$.)

\section{Stable COLLECTIVE BEHAVIORS BEyOND SYNCHRONOUS AND ASYNCHRONOUS BEHAVIORS}

Piecewise linear oscillators exhibit a variety of collective behaviors. For high values $\underline{x}(\bar{\theta} \approx 0)$, the asynchronous state is stable, as for oscillators with a dichotomic behavior. When $\underline{x}$ is decreased under a critical value, the system undergoes a supercritical Hopf bifurcation and the flux oscillates under the influence of the second (unstable) mode (Fig. 4 A). A similar behavior was described in [13], [14] for LIF oscillators with delayed transmission. For $\underline{x} \approx-\bar{x}(\bar{\theta} \approx \pi)$, there is no stable limit cycle so that the unstable mode strongly affects the behavior. The (second) unstable mode grows exponentially and the flux tends to a series of Dirac functions, characterized by two Dirac functions per period (Fig. 4 B). The oscillators do not uniformly spread over the 
circle but gather in two clusters. (The number of clusters is determined by the number $n$ of the unstable mode.)


Fig. 4. A. Near the Hopf bifurcation, the flux is periodic (left) and the oscillators spread over the whole circle, but with two high density regions (right). B. For $\bar{x} \approx-\underline{x}$, the flux is a series of Dirac functions, with two Dirac functions per oscillator period $(T \approx 0.9)$ (left) and the oscillators gather in two clusters (right). (The simulations correspond to the finite population model, with a (large) number of $N=100$ oscillators, and with the parameters $S=1, K=0.510^{-2}, \bar{x}=1$, and $\underline{x}=-0.6$ (A) or $\underline{x}=-0.8(\mathrm{~B}))$

\section{CONCLUSION}

Pulse-coupled integrate-and-fire oscillators are (biologically motivated) models characterized by an interesting dichotomic behavior. The dichotomic behavior is well understood in the original LIF model [2], [3], [9] but even a local stability analysis seems intricate for more general models, including the important QIF model.

In the present paper, we have shown that a local stability analysis is more tractable in the continuum limit, in particular under the assumption of weak coupling. We characterized the stability of quadratic models and we proved the conjecture which still prevails for finite populations. In addition, we showed that the continuum approximation is relevant since we recover known results: finite and infinite populations exhibit a similar dichotomic behavior under identical assumptions.

The recent advances presented in this paper are encouraging results. To face the complex problems arising from the study of pulse-coupled oscillators, considering infinite populations is a relevant approach.

\section{ACKNOWLEDGMents}

This paper presents research results of the Belgian Network DYSCO (Dynamical Systems, Control, and Optimization), funded by the Interuniversity Attraction Poles Programme, initiated by the Belgian State, Science Policy Office. The scientific responsibility rests with its authors. The first author holds a FNRS (Belgian National Fund for Scientific Research) fellowship.

\section{APPENDIX}

Following similar lines as in [12], [14], we linearize the PDE (6) and we derive the eigenvalue equation (11). Given (12), the phase dynamics (8) is rewritten as

$$
\dot{\vartheta}=\frac{d \vartheta}{d \theta} v(\theta, t)=2 \pi J^{*}+\left(J_{0}-J^{*}\right) K \Gamma(\vartheta) .
$$

Defining the density $\rho_{\vartheta}$ so that $\rho_{\vartheta} d \vartheta=\rho d \theta$, one has

$$
J=\dot{\vartheta} \rho_{\vartheta} .
$$

Next, considering the small variations $j=J-J^{*}$ (and $\left.j_{0}=J_{0}-J^{*}\right)$ around the stationary solution, (18)-(19) are linearized and the density expresses as

$$
\rho_{\vartheta}=\frac{1}{2 \pi}+\frac{1}{2 \pi J^{*}} j-\frac{K}{4 \pi^{2} J^{*}} \Gamma(\vartheta) j_{0} .
$$

The continuity equation

$$
\frac{\partial \rho_{\vartheta}}{\partial t}=-\frac{\partial J}{\partial \vartheta}
$$

is equivalent to (6). Using (20), the equation becomes the linear PDE

$$
\frac{\partial j}{\partial t}=-2 \pi J^{*} \frac{\partial j}{\partial \vartheta}+\frac{K}{2 \pi} \Gamma(\vartheta) \frac{d j_{0}}{d t} .
$$

In order to compute the eigenspectrum of the equation, an exponential solution $j(\vartheta, t)=\bar{j}(\vartheta) \exp (\lambda t)$ is injected in (21). Solving the linear differential equation for a mode $\bar{j}$, one obtains

$$
\begin{aligned}
\bar{j}(\vartheta)= & \bar{j}(0) e^{-\lambda \vartheta /\left(2 \pi J^{*}\right)} \\
& \times\left(1+\frac{K \lambda}{4 \pi^{2} J^{*}} \int_{0}^{\vartheta} \Gamma\left(\vartheta^{\prime}\right) e^{\lambda \vartheta^{\prime} /\left(2 \pi J^{*}\right)} d \vartheta^{\prime}\right) .
\end{aligned}
$$

Finally, evaluating (22) in $\vartheta=2 \pi$ and using the boundary condition (7) yield the eigenvalue equation (11).

\section{REFERENCES}

[1] C. S. Peskin, Mathematical Aspects of Heart Physiology. Courant Institute of Mathematical Sciences, New York University, New York, 1975.

[2] R. E. Mirollo and S. H. Strogatz, "Synchronization of pulse-coupled biological oscillators," Siam Journal On Applied Mathematics, vol. 50, no. 6, pp. 1645-1662, Dec. 1990.

[3] A. Mauroy and R. Sepulchre, "Clustering behaviors in networks of integrate-and-fire oscillators," Chaos, vol. 18, no. 3, p. 037122, Sep. 2008, see also the erratum in Chaos 19 (2009), 049902.

[4] E. Izhikevich, Dynamical Systems in Neuroscience: The Geometry of Excitability and Bursting. The MIT press, 2007.

[5] A. Mauroy, J. M. Hendrickx, A. Megretski, and R. Sepulchre, "Global analysis of firing maps," in Proceedings of the 19th International Symposium on Mathematical Theory of Networks and Systems, July 2010, pp. 1775-1782.

[6] B. W. Knight, "Dynamics of encoding in a population of neurons." $J$. gen physiol, vol. 59, no. 6, pp. 734-766, 1972.

[7] A. Winfree, The geometry of biological time. New York: SpringlerVerlag, 2001 (Second Edition).

[8] E. Brown, J. Moehlis, and P. Holmes, "On the phase reduction and response dynamics of neural oscillator populations," Neural Computation, vol. 16, no. 4, pp. 673-715, Apr. 2004.

[9] A. Mauroy and R. Sepulchre, "Global analysis of a continuum model for pulse-coupled oscillators," http://arxiv.org/abs/1102.4511, to be resubmitted. [Online]. Available: http://arxiv.org/abs/1102.4511

[10] G. B. Ermentrout and N. Kopell, "Parabolic bursting in an excitable system coupled with a slow oscillation," Siam Journal On Applied Mathematics, vol. 15, pp. 3457-3466, 1986.

[11] H. S. Carslaw, Introduction to the theory of Fourier's series and integrals. Dover, 1983.

[12] L. F. Abbott and C. van Vreeswijk, "Asynchronous states in networks of pulse-coupled oscillators," Physical Review E, vol. 48, pp. 14831490, 1993.

[13] Y. Kuramoto, "Collective synchronization of pulse-coupled oscillators and excitable units," Physica D, vol. 50, no. 1, pp. 15-30, May 1991.

[14] C. van Vreeswijk, "Partial synchronization in populations of pulsecoupled oscillators," Physical Review E, vol. 54, pp. 5522-5537, 1996. 